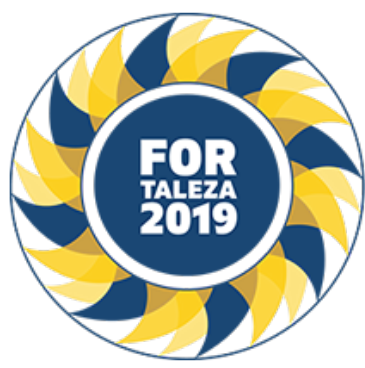

\title{
RETROSPECTIVE ANALYSIS OF 43 CASES OF IDIOPATHIC UVEITIS IN A TERTIARY CENTER
}

Renata Lopes Franscisco Andrade (Unifesp, São Paulo, SP, Brasil), Annelyse de Araújo Pereira (Unifesp, São Paulo, SP, Brasil), Melissa Mariti Fraga (Unifesp, São Paulo, SP, Brasil), Yuslay Fernández Zamora (Unifesp, São Paulo, SP, Brasil), Cristina Muccioli (Unifesp, São Paulo, SP, Brasil), Maria Teresa Terreri (Unifesp, São Paulo, SP, Brasil)

\section{BACKGROUND}

Pediatric uveitis is a rare inflammatory ocular disease, accounting for approximately 5 to $10 \%$ of all uveitis. However, it presents a high risk of ocular complications and visual loss, being an important cause of ocular morbidity and severe visual loss in up to $25-33 \%$ of the cases. The non-infectious causes correspond to 69 to $95 \%$ of the uveitis in childhood, JIA corresponds to 41 to $47 \%$, whereas in 28 to $51 \%$ of the cases no causes are detected (idiopathic). In this study, we aim to describe the clinical and epidemiological characteristics of idiopathic uveitis, the main complications and therapeutic regimens.

\section{MATERIALS AND METHODS}

Retrospective analysis based on chart review of 43 patients with idiopathic uveitis at the Pediatric Rheumatology outpatient clinic. The ophthalmologic evaluation was performed by a specialized ophthalmologist using a slit lamp with biomicroscopy.

\section{RESULTS}

We evaluated 43 patients(a total of 86 eyes), 28 (65.1\%) were female, mean age at the diagnosis was 7.3 years. Regarding to the clinical course of uveitis, 28 patients had a chronic follow up (65.1\%), 13 had recurrent uveitis (30.2\%) and 2 had an acute presentation (4.7\%). In relation to local of uveitis, 24 patients $(55.8 \%)$ presented anterior uveitis, 16 patients (37.2\%) intermediate and $3(7 \%)$ panuveitis. FAN positive was identified in 20 (46.5\%) patients and only one patient had rheumatoid factor positive (2.3\%). Thirty eight patients required DMARDs to control the activity, in a total of 47 synthetic DMARDs. Methotrexate was the first option in 37 cases (86\%) followed by cyclosporine, leflunomide and azathioprine. The biological treatment was used in 8 refractory cases in a total of 13 biological agents. Adalimumab was the first option in 5 cases (62.5\%). Complications were observed in 64 eyes and keratopathy was the most frequent $(28.1 \%)$, followed by cataract $(25 \%)$, presence of synechiae $(14.1 \%)$ and glaucoma / ocular hypertension (10.9\%).

\section{CONCLUSION}

We concluded that idiopathic uveitis presented frequently complications, recurrence/ chronicity and treatment refractoriness. 